\title{
Endoscopic ultrasound-guided hepaticoduodenostomy with anterograde stenting for recurrent hepatic hilar obstruction
}

We present the case of a 52-year-old woman who underwent endoscopic ultrasound-guided hepaticoduodenostomy (EUS-HDS) with anterograde stenting. She had undergone side-by-side stent drainage (the left hepatic and anterior bile ducts) for Bismuth type II hilar bile duct obstruction (HBO) caused by intrahepatic cholangiocarcinoma. She was diagnosed with cholangitis in the posterior bile duct (B6) due to stent occlusion 2 months later. Endoscopic retrograde cholangiopancreatography was performed, but the catheter could not be inserted into B6 (\$ Fig.1). Although balloon dilation of the stent mesh was performed, even the thinnest (5.7 Fr) uncovered self-expandable metal stent (UCSEMS) could not be inserted. Therefore, we performed anterograde stenting via EUS-HDS.

The EUS scope was inserted into the descending duodenum. The B6 duct was identified and punctured with a $19 G$ needle. A 0.025-inch guidewire was inserted from B6 into the common bile duct $(\mathrm{CBD})$ through the mesh of the previous UCSEMS. The fistula site was dilated with a 4-mm balloon. A UCSEMS $(8 \mathrm{~mm} \times 6 \mathrm{~cm}$; ZEOSTENT; Zeon Medical, Tokyo, Japan) was deployed from $B 6$ to the CBD using anterograde stenting. Additionally, a fully covered SEMS ( $8 \mathrm{~mm} \times 8 \mathrm{~cm}$; X-SUIT NIR; Olympus, Tokyo, Japan) was deployed at the fistula site. Finally, cholangiography was performed and confirmed the passage of contrast from B6 via the CBD to the duodenum ( $\vee$ Fig. 2 ; $\triangleright$ Video 1 ).

Post-procedure computed tomography revealed that the intrahepatic bile duct dilatation had disappeared ( $\mathbf{F i g . 3}$ ). There were no procedure-related complications. Clinically, with the cholangitis improved, the patient was able to resume her chemotherapy.

Reintervention after SEMS deployment for $\mathrm{HBO}$ is difficult because it is per-
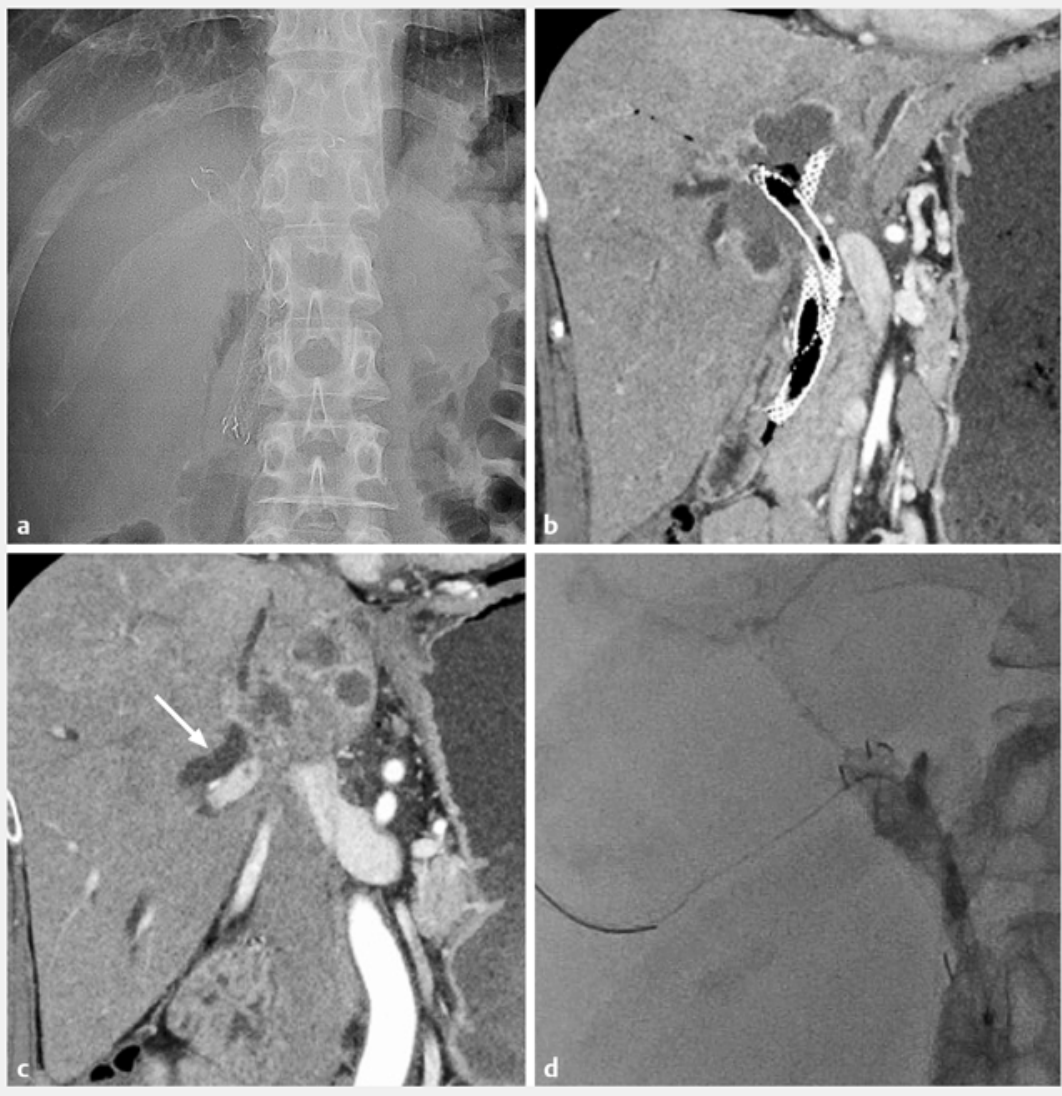

- Fig. 1 Images showing: a, b the appearance of the uncovered self-expandable metal stents that had been previously deployed into the hilar biliary obstruction using the side-by-side method; $\mathbf{c}$ dilatation of the B6 duct; $\mathbf{d}$ the guidewire inserted into the B6 duct during endoscopic retrograde cholangiopancreatography; however, the catheter and stent could not then be inserted.

formed after multi-stenting with a UCSEMS. EUS-HDS [1-3] is an alternative drainage route for $\mathrm{HBO}$; however, there were no reports on EUS-HDS with anterograde stenting. This is the first report demonstrating the usefulness of EUSHDS with anterograde stenting as a rescue drainage procedure for $\mathrm{HBO}$ cases wherein reintervention is difficult.

Endoscopy_UCTN_Code_CPL_1AL_2AD
Acknowledgment

We thank Mr. Yuya Kanai for the help in creating the figures.

\section{Funding}

This work was supported in part by The National Cancer Center Research and Development Fund (31-A-13) and by a grant from The Japanese Foundation for Research and Promotion of Endoscopy (JFE). 

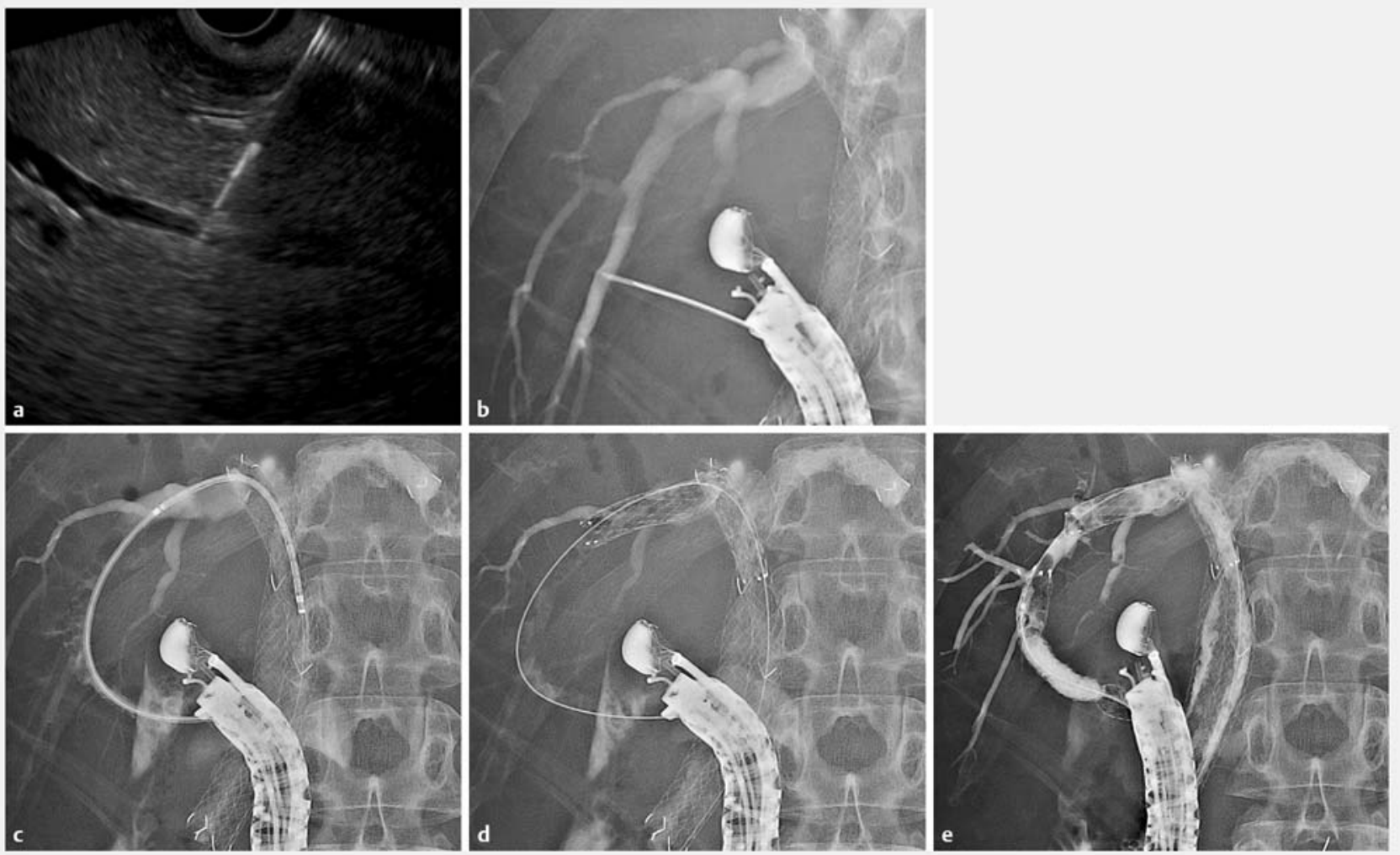

- Fig. 2 Endoscopic ultrasound-guided hepaticoduodenostomy (EUS-HDS) with anterograde stenting showing: a the posterior branch (B6) clearly visualized by EUS from the descending duodenum; $\mathbf{b}$ puncture of the B6 duct with a 19G needle being confirmed by cholangiogram; $\mathbf{c}, \mathbf{d}$ the fistula site being dilated, with a guidewire passed between the mesh of an uncovered self-expandable metal stent (UCSEMS) in the B8 duct, before a UCSEMS is deployed from the B6 branch to the common bile duct using the stent-in-stent method; e cholangiography to confirm the flow of contrast from B6 via the common bile duct to the duodenum, passing through the fully covered SEMS deployed at the fistula site.

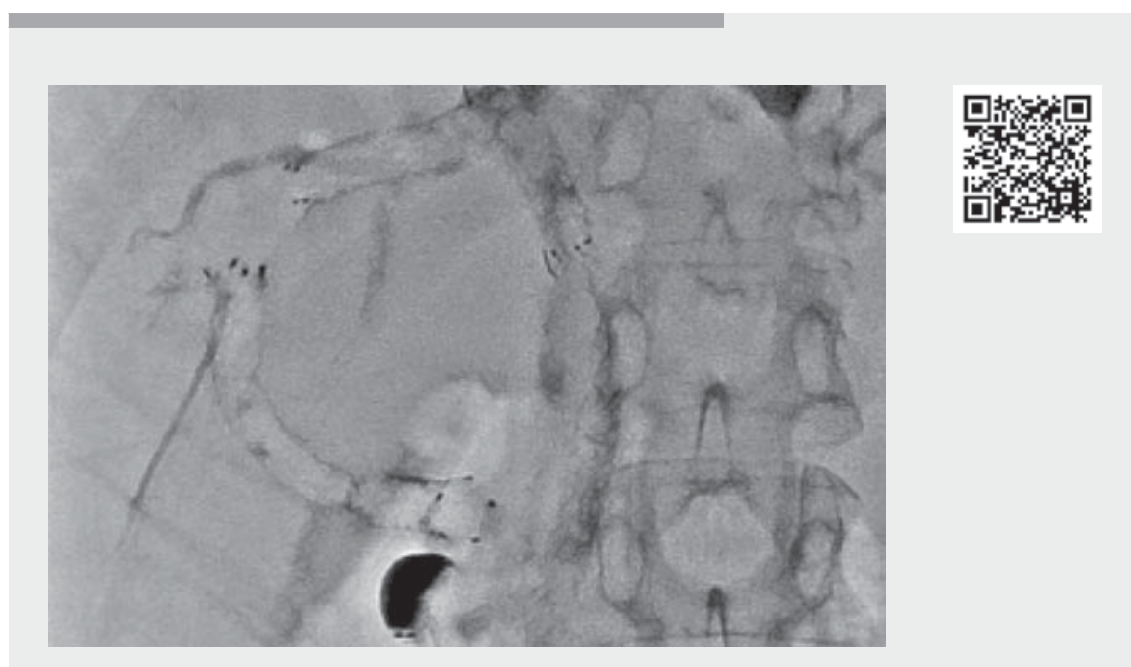

Video 1 Video showing a novel reintervention method for hilar biliary obstruction using endoscopic ultrasound-guided hepaticoduodenostomy with anterograde stenting.

\section{Competing interests}

The authors declare that they have no conflict of interest.

The authors

Shota Harai ${ }^{1}$, Susumu Hijioka ${ }^{1}$, Yuta Maruki ${ }^{1}$, Akihiro Ohba ${ }^{1}$, Yoshikuni Nagashio ${ }^{1}$, Takuji

Okusaka $^{1}$, Yutaka Saito ${ }^{2}$

1 Department of Hepatobiliary and Pancreatic Oncology, National Cancer Center Hospital, Tokyo, Japan

2 Endoscopy Division, National Cancer Center Hospital, Tokyo, Japan

\section{Corresponding author}

\section{Susumu Hijioka, MD}

Department of Hepatobiliary and Pancreatic Oncology, National Cancer Center Hospital, 5-1-1 Tsukiji, Chuo-ku, Tokyo, Japan shijioka@ncc.go.jp 

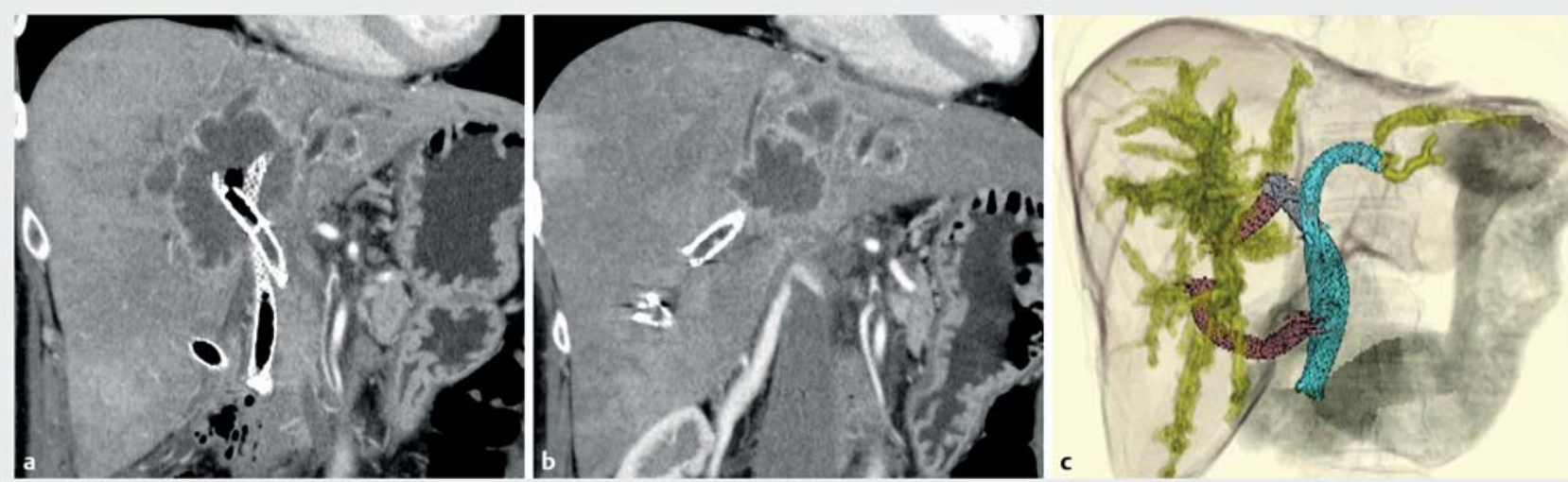

- Fig. 3 Computed tomography (CT) images after endoscopic ultrasound-guided hepaticoduodenostomy (EUS-HDS) showing: a the coronal view; b the sagittal view; c a 3 D construction using a work station (Ziostation2; Ziosoft Inc., Tokyo, Japan).

\section{References}

[1] Hijioka S, Sakamoto Y, Ohba A et al. Novel simultaneous endoscopic ultrasound-guided hepaticoduodenostomy and hepaticogastrostomy for recurrent hepatic hilar obstruction. Endoscopy 2018; 50: e320-e322

[2] Ma KW, So H, Cho DH et al. Durability and outcome of endoscopic ultrasound-guided hepaticoduodenostomy using a fully covered metal stent for segregated right intrahepatic duct dilatation. J Gastroenterol Hepatol 2020; 35: 1753-1760

[3] Hori Y, Hayashi K, Naitoh I et al. Combined transpapillary drainage and endoscopic ultrasound-guided hepaticoduodenostomy after failed manipulation under cholangioscopy guidance. Endoscopy 2020. doi:10.1055/a-1216-1254

\section{Bibliography}

Endoscopy 2022; 54: E398-E400

DOI 10.1055/a-1559-1550

ISSN 0013-726X

published online 27.8.2021

(c) 2021. Thieme. All rights reserved.

Georg Thieme Verlag KG, Rüdigerstraße 14, 70469 Stuttgart, Germany

\section{ENDOSCOPY E-VIDEOS \\ https://eref.thieme.de/e-videos}

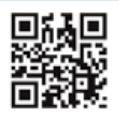

Endoscopy E-Videos is an open access online section, reporting on interesting cases and new techniques in gastroenterological endoscopy. All papers include a high quality video and all contributions are freely accessible online. Processing charges apply (currently EUR 375), discounts and wavers acc. to HINARI are available.

This section has its own submission website at

https://mc.manuscriptcentral.com/e-videos 